\title{
Can CAP guideline adherence improve patient outcome in internal medicine departments?
}

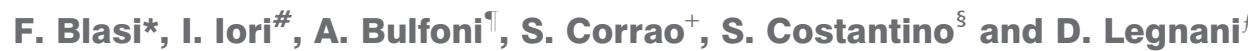

ABSTRACT: The impact of compliance with Italian guidelines on the outcome of hospitalised community-acquired pneumonia (CAP) in internal medicine departments was evaluated.

All Fine class IV or V CAP patients were included in this multicentre, interventional, before-andafter study, composed of three phases: 1) a retrospective phase (RP; 1,443 patients); 2) a guideline implementation phase; and 3) a prospective phase (PP; 1,404 patients).

Antibiotic prescription according to the guidelines increased significantly in the PP. The risk of failure at the end of the firstline therapy was significantly lower in the PP versus the RP (odds ratio

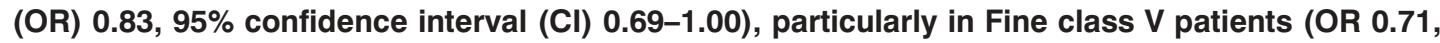
$95 \% \mathrm{Cl}$ 0.51-0.98). Analysis of outcome in the overall population (2,847 patients) showed a statistically significant advantage for compliant versus noncompliant therapies in terms of failure rate (OR $0.74,95 \% \mathrm{Cl} 0.60-0.90)$ and an advantage in terms of mortality (OR $0.77,95 \% \mathrm{Cl} 0.58-$ 1.04). Antipneumococcal cephalosporin monotherapy was associated with a low success rate (68.6\%) and the highest mortality (16.2\%); levofloxacin alone and the combination of cephalosporin and macrolide resulted in higher success rates $(79.1$ and $76.7 \%$, respectively) and significantly lower mortalities (9.1 and 5.7\%, respectively).

Overall, a low compliance with guidelines in the prospective phase (44\%) was obtained, indicating the need for future more aggressive and proactive approaches.

\section{KEYWORDS: Antibiotic, community-acquired pneumonia, guidelines}

$\mathrm{n}$ Europe, the overall incidence of communityacquired lower respiratory tract infections

(LRTIs) was found to be 44 cases per 1,000 population per year in a single general practice. However, the incidence was two- to four-times higher in people aged $\geqslant 60$ yrs than in those aged $<50$ yrs [1]. A study in Finland of 546 patients with community-acquired pneumonia (CAP) found that the overall incidence was 11.6 per 1,000 inhabitants, $37 \%$ of the patients were aged $<15 \mathrm{yrs}, 31 \%$ were aged $\geqslant 60 \mathrm{yrs}, 42 \%$ were admitted to hospital and the case fatality rate was $4 \%$ [2]. On average, the mortality rate in patients with CAP who have been hospitalised is $\sim 12 \%$ in both the USA and Europe, while in outpatients the mortality rate is lower, at $\sim 5 \%$. As expected, patients with more-severe CAP have a higher mortality rate of $29 \%$. The highest death rates $(40 \%)$ were found in those who are admitted to an intensive care unit (ICU).
Recent guidelines have provided a rational framework for empirical antibiotic use based on epidemiological criteria [3,4]. Mortality risk may be assessed through the use of clinical prediction scores such as the Fine classification [5]. It is generally recognised that patients who fall into the upper spectrum of severity (Fine classes IV and $\mathrm{V}$ ) require hospitalisation.

Although some of the guidelines were evidence based, there is limited evidence to support the recommendations regarding antimicrobial therapy and only a few studies have addressed the validation of guidelines [6, 7]. In the present study, the primary objective was to evaluate the impact of compliance to a new set of Italian guidelines on the management of patients with CAP, hospitalised in internal medicine (IM) departments.

\section{AFFILIATIONS}

*Institute of Respiratory Diseases, University of Milan, Maggiore Hospital, Fondazione IRCCS

Policlinico, Mangiagalli e Regina Elena,

${ }^{f}$ Institute of Respiratory Diseases, University of Milan, "L. Sacco" Hospital, Milan,

\#Dept of Internal Medicine, Azienda Ospedaliera Arcispedale Santa Maria Nuova, Centro Studi FADOI, Reggio Emilia,

"Internal Medicine Unit, Azienda Ospedaliero-Universitaria Santa Maria della Misericordia di Udine, Centro Studi FADOI, Udine, ${ }^{+}$Biomedical Dept of Internal Medicine, University of Palermo,

Centro studi FADOI, Palermo, and

${ }^{\S}$ Dept of Internal Medicine, University Campus Bio-Medico, Centro studi

FADOI, Rome, Italy.

CORRESPONDENCE

F. Blasi, Istituto Malattie

dell'Apparato Respiratorio, University of Milan, Padiglione Sacco Ospedale Maggiore Fondazione IRCCS Policlinico, Mangiagalli e Regina Elena, Via F. Sforza 35, 20122 Milan, Italy.

Fax: 390250320628

E-mail: francesco.blasi@unimi.it

Received:

July 222007

Accepted after revision:

May 082008

\section{STATEMENT OF INTEREST}

Statements of interest for F. Blasi, I. Iori, A. Bulfoni, S. Corrao and D. Legnani, and for the study itself can be found at www.erj.ersjournal. $\mathrm{com} / \mathrm{misc} /$ statements.shtml 


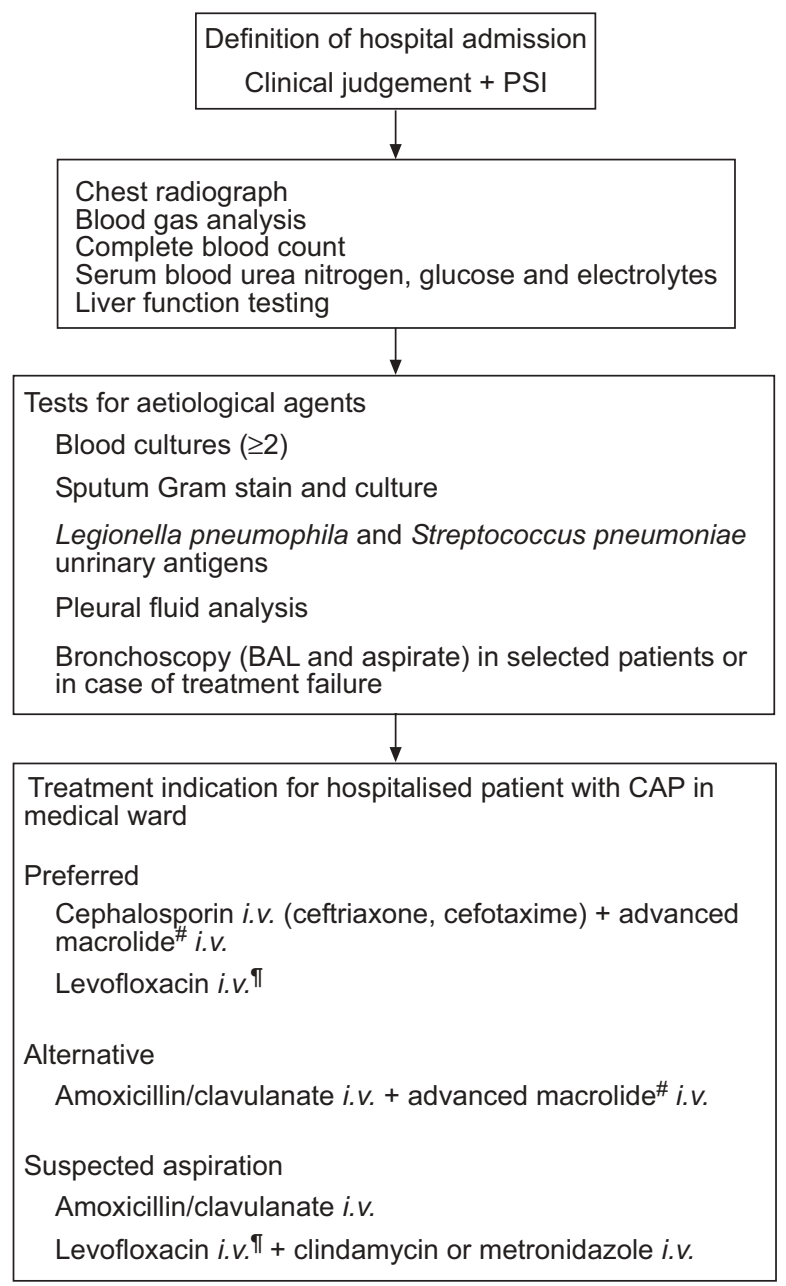

FIGURE 1. Italian Federation of Internal Medicine (FADOI) guidelines' indications for initial management of hospitalised community-acquired pneumonia (CAP). PSI: pneumonia severity index; BAL: bronchoalveolar lavage. *: azithromycin or clarithromycin; ": levofloxacin is the only fluoroquinolone with approved intravenous formulation in Italy.

\section{MATERIAL AND METHODS}

\section{Patient characteristics}

All patients with CAP classified as Fine class IV or V (excluding those in Fine $\mathrm{V}$ directly admitted to the ICU) were included in the study [5].

CAP was defined as an acute LRTI characterised by 1) an acute pulmonary infiltrate evident on chest radiographs and compatible with pneumonia, and 2) confirmatory findings on clinical examination and acquisition of the infection in the community [4]. Immunocompromise was defined as primary immunodeficiency, immunodeficiency secondary to radiation treatment or use of cytotoxic drugs or steroids (daily doses of $>20 \mathrm{mg}$ of prednisolone or the equivalent for 12 weeks), or AIDS [8, 9]. Pre-existing chronic obstructive pulmonary disease was diagnosed using criteria reported elsewhere [10].

The study was approved by the local ethic committee/review board and informed consent was obtained for each eligible

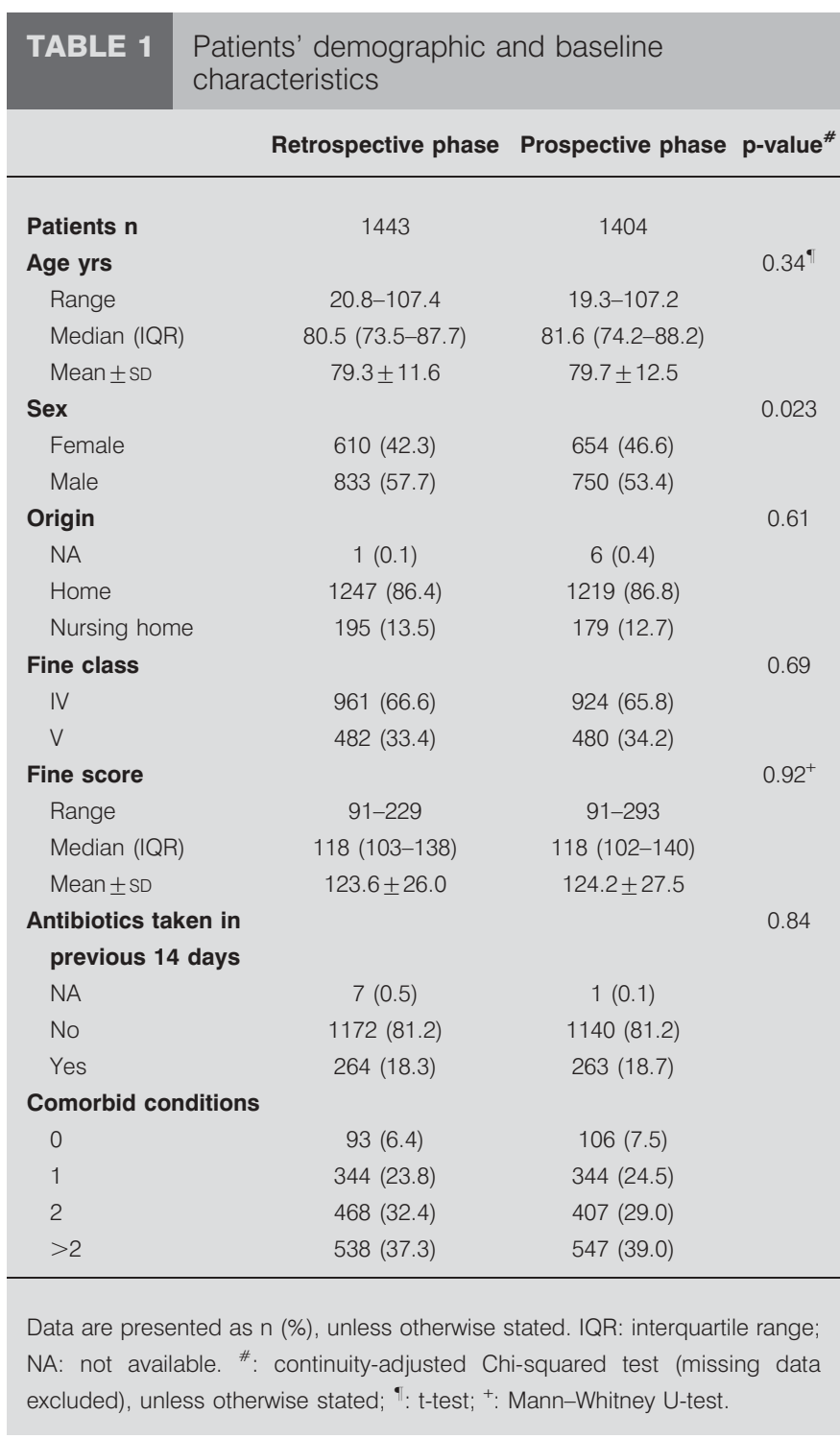

subject prior to entry into the prospective phase (PP). Patients did not receive any new investigational drug or innovative diagnostic procedure and disease management was performed according to the usual clinical practice of the centre.

\section{Study design}

The present study was an Italian, national, multicentre $(n=31)$ interventional, before-and-after survey. The intervention was the implementation of Italian guidelines on management of CAP in IM departments.

The study was composed of three phases: 1) a retrospective phase (RP), in which each centre retrospectively collected data on diagnosis and management of all patients with CAP admitted to the IM departments between January 1 and December 31, 2002, according to local guidelines/clinical practice; 2) introduction and implementation of national CAP guidelines; and 3) the PP, in which each centre prospectively enrolled all patients hospitalised with CAP, between June 6, 2003 and May 31, 2004, and collected clinical and disease management data. 


\begin{tabular}{|c|c|c|c|}
\hline \multirow[t]{2}{*}{ TABLE 2} & \multicolumn{3}{|c|}{$\begin{array}{l}\text { Initial therapy, according to compliance with } \\
\text { therapeutic Italian Federation of Internal } \\
\text { Medicine recommendations and to study phase }\end{array}$} \\
\hline & $\begin{array}{c}\text { Retrospective } \\
\text { phase }\end{array}$ & $\begin{array}{c}\text { Prospective } \\
\text { phase }\end{array}$ & $p$-value \\
\hline \multicolumn{4}{|l|}{ All patients } \\
\hline Subjects n & $1443(100)$ & $1404(100)$ & \\
\hline \multicolumn{4}{|l|}{ Compliant } \\
\hline Total & $476(33.0)$ & $616(43.9)$ & $<0.001^{\#}$ \\
\hline Monotherapy & $150(10.4)$ & $214(15.2)$ & \\
\hline Combination & 326 (22.6) & 402 (28.6) & \\
\hline \multicolumn{4}{|l|}{ Noncompliant } \\
\hline Total & 967 (67.0) & $788(56.1)$ & \\
\hline Monotherapy & $710(49.2)$ & $551(39.2)$ & \\
\hline Combination & 257 (17.8) & 237 (16.9) & \\
\hline \multicolumn{4}{|l|}{ Fine class IV } \\
\hline Subjects n & $961(100)$ & $924(100)$ & \\
\hline \multicolumn{4}{|l|}{ Compliant } \\
\hline Total & 323 (33.6) & $421(45.6)$ & $<0.001^{\#}$ \\
\hline Monotherapy & 101 (10.5) & $142(15.4)$ & \\
\hline Combination & 222 (23.1) & $279(30.2)$ & \\
\hline \multicolumn{4}{|l|}{ Noncompliant } \\
\hline Total & 638 (66.4) & $503(54.4)$ & \\
\hline Monotherapy & 470 (48.9) & 350 (37.9) & \\
\hline Combination & 168 (17.5) & $153(16.6)$ & \\
\hline \multicolumn{4}{|l|}{ Fine class V } \\
\hline Subjects n & $482(100)$ & $480(100)$ & \\
\hline \multicolumn{4}{|l|}{ Compliant } \\
\hline Total & $153(31.7)$ & $195(40.6)$ & $<0.01^{\natural}$ \\
\hline Monotherapy & 49 (10.2) & $72(15.0)$ & \\
\hline Combination & $104(21.6)$ & $123(25.6)$ & \\
\hline \multicolumn{4}{|l|}{ Noncompliant } \\
\hline Total & $329(68.3)$ & $285(59.4)$ & \\
\hline Monotherapy & $240(49.8)$ & $201(41.9)$ & \\
\hline Combination & 89 (18.5) & $84(17.5)$ & \\
\hline
\end{tabular}

Data are presented as $\mathrm{n}(\%)$, unless otherwise stated. \#: both continuityadjusted Chi-squared test and Wald Chi-squared test (logistic regression adjusting for study centre as a random effect); " : continuity-adjusted Chisquared test, $p=0.005$ and Wald Chi-squared test, $p=0.003$.

The study was designed by F. Blasi and D. Legnani, with input from an advisory board that included all authors (see the Acknowledgements section).

\section{Guideline implementation}

During late 2001 and early 2002, a multidisciplinary group of experts prepared a new set of guidelines for the management of LRTIs, including CAP, in the IM setting on behalf of the Italian Federation of Internal Medicine (FADOI) [11]. Guideline implementation began after publication, between December 2002 and June 2003. The publication was distributed to all FADOI study centres, and the guidelines were presented at the Italian Internal Medicine national congress and discussed in ad hoc investigator groups prior to activation of the PP. Figure 1 summarises the general management and treatment indications suggested by the FADOI treatment guidelines [11].

\section{Data collection}

Data extracted from the clinic registries and patient records were used. The total numbers of CAP admissions in hospital and IM departments in each study period were also collected.

Patients' demographics, history, clinical status and diagnosis were collected at baseline on a case report form (CRF). Initial empirical antibiotic therapy and outcome were recorded. Information on initial antibiotic, dose, frequency and duration of administrations was also collected. All changes to the initial antimicrobial therapy were recorded. Antibiotic therapy assessment included therapy outcome (success or failure) and reason for failure (death, referral to ICU, intolerance, therapeutic failure or lack of efficacy). In case of failure, the second antibiotic therapy cycle was also recorded.

Clinical success was defined as the resolution or improvement of the symptoms of pneumonia, at the end of the first cycle of therapy. A therapeutic failure was defined as the worsening of symptoms or fever or the need for a new course of antibiotics.

Data regarding aetiology and specimens collected for pathogen isolation were also recorded. The participating sites were locally monitored by ad hoc trained personnel of a contract research organisation (CRO; Fidea srl, Milan, Italy). All CRFs were sent to the biostatistical department of the CRO and double-entered into a computer database. The database was checked for consistency and data that failed were reviewed by the study sponsor (Sanofi-Aventis Italia, Milan, Italy), with input from all authors.

\section{Statistical analysis}

The principal measure of compliance with guideline indications was the change in antibiotic prescription behaviour. The primary efficacy variables were the clinical outcome (success or failure) and mortality rate of the two survey phases at the end of the first therapy cycle. The time to clinical success within 30 days of admission was also examined.

Data were summarised using proportions if categorical, and mean \pm SD or median with quartiles if continuous. Associations between two categorical variables were tested using the Chisquared test, while associations between a categorical and a numerical variable were tested using unpaired t-tests or the Mann-Whitney U-test. Compliance with FADOI treatment recommendations was compared between study phases using both the Chi-squared test and a mixed-effect logistic regression model with the study centre as a random effect.

Analysis of clinical outcome was conducted using mixed-effect logistic regression models including study phase, Fine score in 10-point intervals (91-100 to 121-130, Fine class IV; and 131140 to $>200$, Fine class V) and antibiotic treatment in the previous 2 weeks (yes or no) as fixed effects and study centre as a random effect. Age, sex, origin (home versus nursing home residence) and concomitant diseases were not multivariateindependent predictors as they are accounted for in the Fine score algorithm, although they could be univariately associated with outcomes. This basic model was then extended to include therapy, categorised as compliant or noncompliant to FADOI recommendations, or further divided according to main antibacterial class. In-hospital mortality was analysed in the same manner as clinical outcome; however, models 
TABLE 3 Outcome and mortality at the end of initial therapy, according to study phase

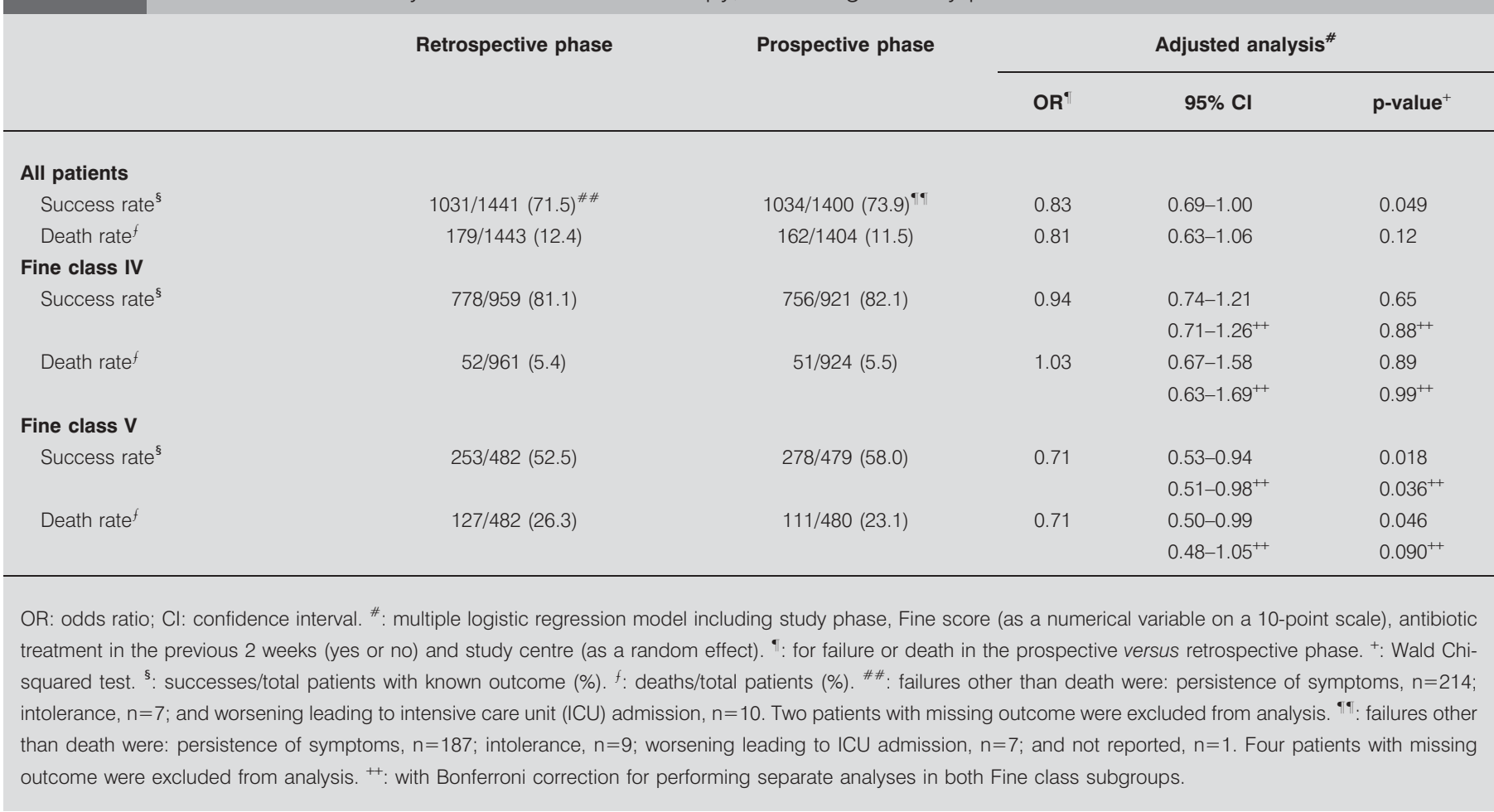

comparing main antibacterial treatments were not adjusted for study centre because of the small number of fatal events per treatment group. According to an intention-to-treat approach, only initial therapies, irrespective of their duration, were considered in all multivariate analyses. Subgroup analyses using similar models were also conducted according to Fine class. Odds ratios (ORs) with confidence intervals (CIs) and Wald-type p-values were calculated, adjusting for all other factors included in the model.

Time to success, i.e. time to discharge, was calculated from admission to discharge after cure using the Kaplan-Meier method. This time was equivalent to the length of hospital stay in instances of success within 30 days of admission, and was otherwise supressed at 30 days, or earlier in instances of failure when the patient was alive (e.g. transferred to the ICU). Comparisons of time to success were carried out using Cox's proportional hazard regression models, including study phase, Fine score, previous antibiotic prescription and initial therapies (compliant versus noncompliant), with study centre as a stratification factor. Hazard ratios (HRs) with CIs and p-values were calculated, provided that the proportionality assumption (tested using time-dependent covariates) was met.

All tests were two-sided. Values of $\mathrm{p}<0.05$ were considered statistically significant; therefore, ORs were reported with their $95 \%$ CI. For analyses of clinical outcome and mortality within subgroups, i.e. Fine class, CIs and p-values with Bonferroni correction (exact formula) for multiple comparisons have been reported.

Statistical analysis was performed by Studio Associato Airoldi, Cicogna e Ghirri (Milan, Italy).

\section{RESULTS}

A total of 1,443 and 1,404 patients were included in the RP and PP of the study, respectively, by the participating IM departments. Among the total number of pneumonia hospital admissions, $24.0 \%$ in the RP and $24.7 \%$ in the PP were admitted to IM departments; of these, $60.0 \%$ in the RP and $60.9 \%$ in the PP were classified as Fine class IV-V patients. Patients' main demographic and clinical characteristics are summarised in table 1. A significant number of elderly patients were admitted, with 74.2 and $70.6 \%$ of patients being aged 65-90 yrs in the RP and PP, respectively, while 16.0 and $19.2 \%$, respectively, were aged $\geqslant 90$ yrs. A total of 66.6 and $33.4 \%$ of patients in the RP, and 65.8 and $34.2 \%$ in the PP, were classified as Fine class IV and V, respectively. One or more comorbidities were present in 93.6 and $92.5 \%$ of patients in the $\mathrm{RP}$ and PP, respectively. Two or more chronic diseases associated with CAP were present in $69.7 \%$ of cases in RP and $67.9 \%$ of cases in PP (table 1 ).

After guideline implementation, compliant antibiotic prescribing increased significantly in the PP compared with the RP (44 versus $33 \%$, respectively; $\mathrm{p}<0.001$ ). The increase in compliance was present in both Fine class IV and V patients (table 2). Specifically, the use of a $\beta$-lactam/ $\beta$-lactamase inhibitor combination with an advanced macrolide nearly doubled during the PP. Moreover, a shift from prescribing $\beta$-lactams to prescribing respiratory fluoroquinolones as initial monotherapy was observed (see the online supplementary data). Mean \pm SD daily drug dosages for the most commonly prescribed antibiotics were as follows: ceftriaxone $1.7 \pm 0.4 \mathrm{~g}$, levofloxacin $0.5 \pm 0.1 \mathrm{~g}$, amoxicillin-clavulanate $4.4 \pm 0.9 \mathrm{~g}$, azithromycin $0.5 \pm 0 \mathrm{~g}$ and clarithromycin $1.0 \pm 0.1 \mathrm{~g}$. 


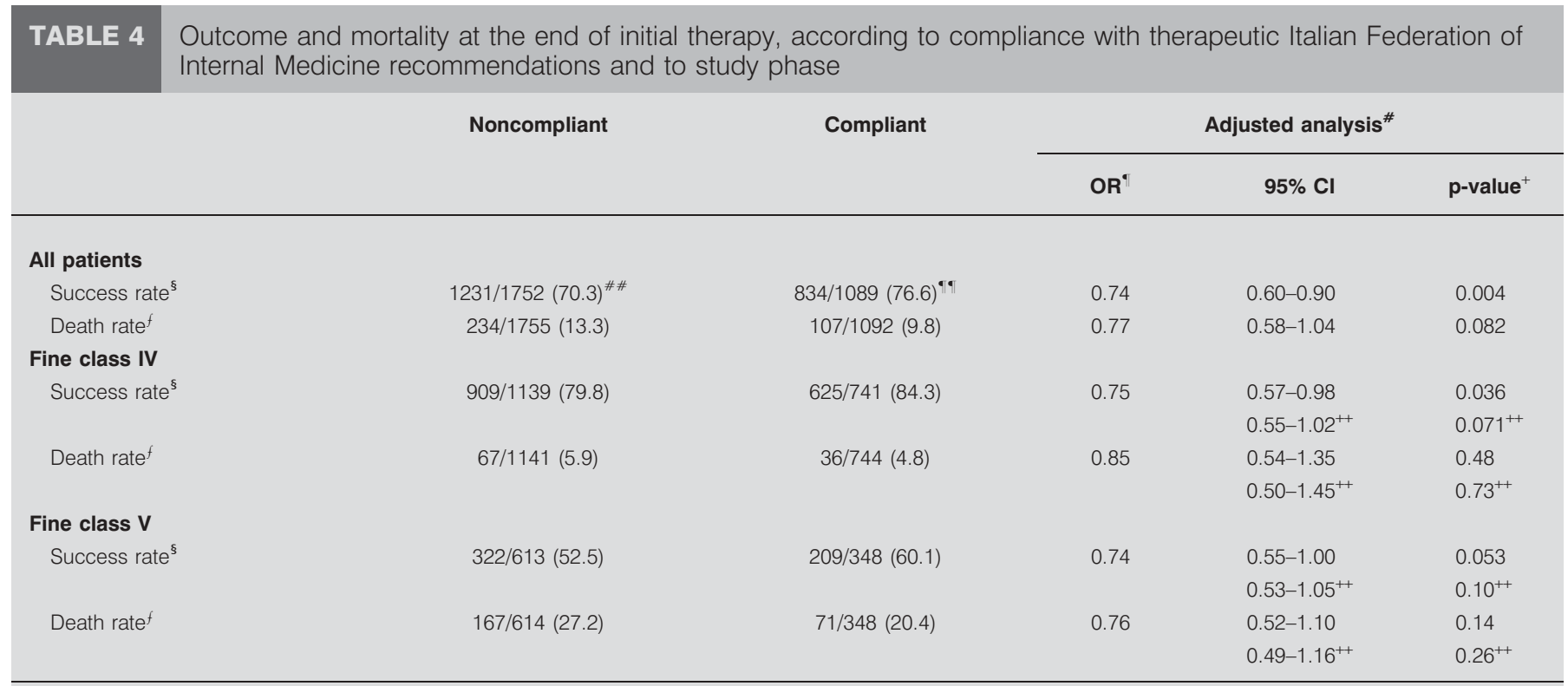

OR: odds ratio; Cl: confidence interval. " ${ }^{\text {: }}$ multiple logistic regression model including compliance with therapeutic recommendations, study phase, Fine score (as a numerical variable on a 10-point scale), antibiotic treatment in the previous 2 weeks (yes or no) and study centre (as a random effect). ": for failure or death in compliant versus noncompliant therapy and in the prospective versus retrospective phase. ${ }^{+}$: Wald Chi-squared test. ${ }^{\varsigma}$ : successes/total patients with known outcome (\%). ${ }^{f}$ : deaths/ total patients (\%). \#\#: failures other than death were: persistence of symptoms, $n=264$; intolerance, $n=8$; worsening leading to intensive care unit (ICU) admission, $\mathrm{n}=14$; and not reported, $\mathrm{n}=1$. Three patients with missing outcome were excluded from analysis. " : failures other than death were: persistence of symptoms, $\mathrm{n}=137$; intolerance, $n=8$; and worsening leading to ICU admission, $n=3$. Three patients with missing outcome were excluded from analysis. ${ }^{++}$: with Bonferroni correction for performing separate analyses in both Fine class subgroups.

Firstline antibiotic therapy was successful in 1,031 (71.5\%) out of 1,441 patients in the RP and in $1,034(73.9 \%)$ out of 1,400 patients in the PP with known outcome. The OR $(95 \% \mathrm{CI})$ for failure in the PP versus the RP, adjusted for risk factors and study centre, was $0.83(0.69-1.00 ; \mathrm{p}=0.049)$. The benefit was particularly evident in Fine class $\mathrm{V}$ patients, in whom the adjusted OR (95\% CI) was 0.71 (0.51-0.98; $p=0.036$ with Bonferroni correction). The concomitant reduction in overall mortality, from $12.4 \%$ in the RP to $11.5 \%$ in the PP, was not statistically significant $(0.81(0.63-1.06) ; \mathrm{p}=0.12$; table 3$)$.

Success rates during firstline antibiotic treatment were 74.5 and $70.1 \%$ in the RP, versus 78.2 and $70.5 \%$ in the PP, for compliant therapies and noncompliant therapies, respectively. Mortality at the end of firstline antibiotic treatment in the RP was $11.1 \%$ for compliant therapies and $13.0 \%$ for noncompliant therapies, versus $8.8 \%$ for compliant therapies and $13.7 \%$ for noncompliant therapies in the PP (see online supplementary data). The interactions between compliance and study phase were not statistically significant $(p>0.10)$. The analysis of outcome and mortality at the end of initial therapy for the overall population $(2,847$ patients in total in the RP and PP), according to compliance with the FADOI treatment guidelines and adjusting for risk factors, study phase and study centre, showed a significant advantage for compliant versus noncompliant therapies in terms of success rate, with an adjusted OR (95\% CI) for failure of $0.74(0.60-0.90 ; p=0.004)$. The effect on mortality failed to achieve statistical significance, although the point estimate was similar (0.77 (0.58-1.04); $\mathrm{p}=0.082$; table 4). In these models, the ORs for failure and for mortality were still in favour of the PP after adjusting for compliance, although to a lesser extent than in models that did not adjust for compliance: the OR $(95 \% \mathrm{CI})$ for failure in the PP versus RP after adjusting for compliance, in addition to risk factors and study centre, was $0.86(0.71-1.03 ; \mathrm{p}=0.10)$, and the OR $(95 \% \mathrm{CI})$ for death was $0.84(0.64-1.10 ; p=0.19)$, compared with ORs of 0.83 and 0.81 for failure and death, respectively, in models that adjusted for risk factors and study centre but not for compliance (see online supplementary data).

These results were mirrored by the adjusted HRs for discharge from hospital within 30 days of admission (fig. 2). Considering the overall population (RP and PP), compliant versus noncompliant therapies resulted in an HR (95\% CI) of $1.10(1.00$ $1.20 ; \mathrm{p}=0.050)$ for an earlier discharge in patients treated according to guidelines (fig. 2a). Figure 2 shows the HRs for discharge in Fine class IV and V (fig. $2 \mathrm{~b}$ and c, respectively).

Table 5 shows the results of considering the efficacy of different antibiotics, in terms of success rate and adjusted ORs. Comparing the different antibiotics with antipneumococcal cephalosporin monotherapy, for which the success rate was $68.6 \%$, levofloxacin alone was the most active (success rate $79.1 \%$; OR 0.65 (95\% CI $0.45-0.95) ; p=0.026)$, followed by the combination of levofloxacin and antipneumococcal $\beta$-lactam (78.8\%; $0.66(0.40-1.08) ; p=0.097)$ and by the combination of macrolide and cephalosporins $(76.7 \%$; $0.72(0.49-1.05) ; \mathrm{p}=0.084)$.

Antipneumococcal cephalosporin monotherapy was associated with the highest mortality $(16.2 \%)$. Levofloxacin alone and the combination of cephalosporin and macrolide resulted in a significantly lower mortality (9.1 and 5.7\%, respectively), whereas the combination of antipneumococcal penicillin and 
macrolide had no significant difference in mortality when compared with cephalosporin alone (fig. 3).

When the present study commenced and according to the existing guidelines, nursing home patients were included as CAP. Being a nursing home resident is acknowledged as a risk factor in the Fine scoring system by assigning 10 additional points. Therefore, differences in the proportion of nursing home patients were accounted for in the analyses of the outcomes (13.5 and $12.8 \%$ in the RP and PP, respectively, $\mathrm{p}=0.61 ; 11.3$ and $14.3 \%$ for compliant and noncompliant therapies, respectively, $\mathrm{p}=0.023$ ). However, a sensitivity analysis was performed, excluding patients coming from nursing homes (see online supplementary data). The OR (95\% CI) for mortality of the PP versus RP was slightly lower, at $0.80(0.59-1.09)$ instead of 0.81 , while for failure it was increased from 0.83 to $0.86(0.70-1.05)$ and the corresponding $\mathrm{p}$-value increased from 0.049 to 0.14 . The ORs $(95 \% \mathrm{CI})$ of compliant versus noncompliant therapies were lower than in the main analysis: $0.72(0.58-0.89)$ instead of 0.74 for failure, 0.74 (0.53-1.03) instead of 0.77 for mortality, the corresponding $\mathrm{p}$-values being similar to the whole-sample analysis (see online supplementary data).

A significant association was found between increasing Fine score and CAP mortality, with an adjusted OR (95\% CI) of 1.43 $(1.36-1.50 ; \mathrm{p}<0.0001)$ for each 10 -unit increase over the whole range of values (91 to $>200$ ). In addition, a significant association was identified between increasing Fine score and clinical failure, with an adjusted OR (95\% CI) of 1.30 (1.25-1.34; $\mathrm{p}<0.0001$ ) for each 10-unit increase between 91 and $>200$ (see online supplementary data).

Previous antibiotic treatment was positively associated with clinical failure, with an adjusted OR (95\% CI) of 1.46 (1.16-1.84; $\mathrm{p}=0.002)$. Conversely, previous antibiotic treatment was not significantly associated with increased mortality (1.26 (0.911.75); $\mathrm{p}=0.15$; see online supplementary data).

Concerning the aetiological diagnostic tests performed, no difference was observed between the two study phases. A possible aetiological diagnosis was obtained in $<12 \%$ of patients in both phases, mainly from sputum specimen (data not shown).

\section{DISCUSSION}

Notwithstanding the advances in the antimicrobial management of pneumonia, this disease is still associated with considerable morbidity and mortality, especially in elderly patients. Over the last decade, several national and international guidelines have been devised in order to improve the management of pneumonia. Guidelines deal with different aspects of pneumonia management, from site of care to severity criteria, diagnostic tests and empirical antibiotic therapy. So far, few studies have attempted to give an overall picture of pneumonia management in "real life" conditions or evaluated the consequences of disease management following guideline implementation [6,7]. Following the release of the Italian guidelines for pneumonia management in 2002, 31 IM departments from across Italy were enrolled. Centres provided retrospective data on pneumonia management in their wards for 2002, centre personnel were instructed on guideline changes and modifications through ad hoc meetings and
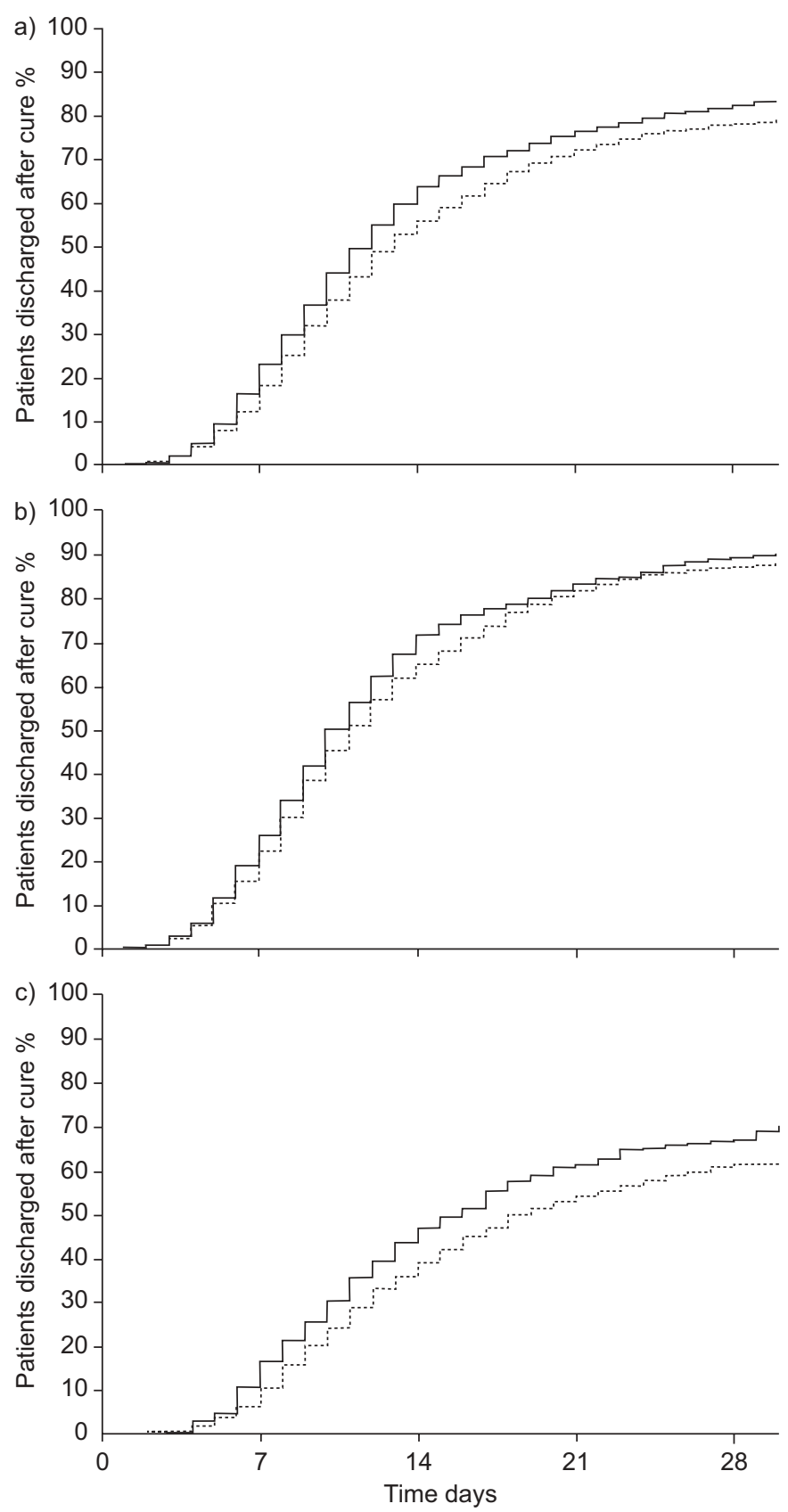

FIGURE 2. Discharge from hospital within 30 days of admission, according to compliance $(-)$ or noncompliance $(\cdots)$ with therapeutic recommendations for a) all patients, b) Fine class IV patients and c) Fine class $V$ patients. Hazard ratios (HRs), the ratios of the probability of being discharged for patients initially treated with compliant therapy versus noncompliant therapy, were calculated using Cox's proportional-hazard regression models adjusting for study phase, Fine score, previous antibacterial treatment and study centre. HRs (95\% confidence intervals) were as follows: a) $1.10(1.00-1.20), p=0.050$; b) 1.08 (0.95-1.22), $p=0.35$ with Bonferroni correction for performing the same comparison in both Fine class subgroups; and c) 1.19 (0.97-1.45), $p=0.11$ with Bonferroni correction for performing the same comparison in both Fine class subgroups.

national congresses, and prospective data on pneumonia management of admitted patients were collected between 2003 and 2004. The present study is the first in Italy to evaluate 
TABLE 5 Outcome at the end of initial therapy, according to type of drug

\begin{tabular}{|c|c|c|c|c|}
\hline \multirow[t]{2}{*}{ Initial therapy } & \multirow[t]{2}{*}{ Success rate ${ }^{\#}$} & \multicolumn{3}{|c|}{ Adjusted analysis" } \\
\hline & & OR $^{+}$ & $95 \% \mathrm{Cl}$ & p-value ${ }^{\mathrm{s}}$ \\
\hline Ceftriaxone, cefotaxime or cefepime alone & $310 / 452(68.6)$ & & & \\
\hline Amoxycillin/clavulanate or ampicillin/sulbactam alone & 268/378 (70.9) & 0.82 & $0.58-1.16$ & 0.25 \\
\hline Levofloxacin alone & 287/363 (79.1) & 0.65 & $0.45-0.95$ & 0.026 \\
\hline Amoxycillin/clavulanate or ampicillin/sulbactam plus macrolide & $168 / 229(73.4)$ & 0.70 & $0.46-1.07$ & 0.093 \\
\hline Levofloxacin plus antipneumococcal $\beta$-lactam $f$ & 123/156 (78.8) & 0.66 & $0.40-1.08$ & 0.097 \\
\hline Noncompliant combinations & $325 / 463(70.2)$ & 0.98 & $0.71-1.36$ & 0.90 \\
\hline
\end{tabular}

OR: odds ratio; Cl: confidence interval. *: successes/total patients on given therapy (\%); ": multiple logistic regression model including initial therapy, study phase, Fine score (as a numerical variable on a 10-point scale), antibiotic treatment in the previous 2 weeks (yes or no) and study centre (as a random effect); ${ }^{+}$: for failure with each therapy versus ceftriaxone, cefotaxime or cefepime alone; ${ }^{\varsigma}$ : Wald Chi-squared test; $f$ : ceftriaxone, cefotaxime, cefepime, amoxicillin/clavulanate or equivalent penicillin

before-and-after clinical practice changes in pneumonia following guideline implementation.

Antibiotic prescribing measures, outcome and habits were compared during the RP and PP. After guideline implementation, a significant increase in the use of compliant therapies occurred; in particular, combination therapy with $\beta$-lactam plus macrolide and a respiratory fluoroquinolone monotherapy was observed, as opposed to monotherapy with $\beta$-lactam. These changes were associated with a significant reduction in the OR for failure following guideline implementation, particularly in

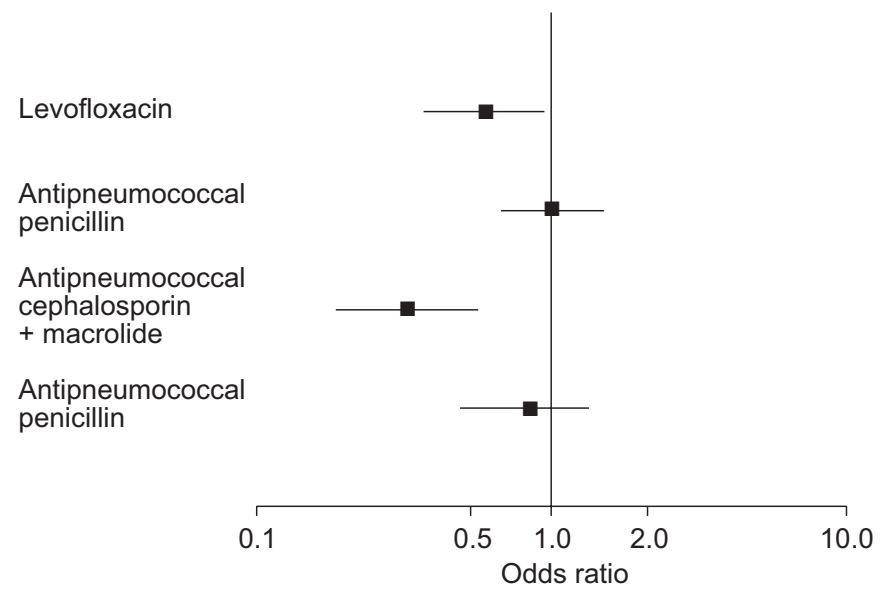

FIGURE 3. Mortality during first therapy cycle according to main initial therapies. Data are presented as adjusted odds ratios (ORs) with 95\% confidence intervals (Cls) versus antipneumococcal cephalosporin alone. Multiple logistic regression model including initial therapy, study phase, Fine score (as a numerical variable on a 10-point scale) and previous antibiotic treatment (yes or no). ORs $(95 \% \mathrm{Cls})$ were as follows: levofloxacin $0.59(0.37-0.94), p=0.026$; antipneumococcal penicillin 1.01 (0.68-1.50), $p=0.97$; antipneumococcal cephalosporin and macrolide $0.32(0.19-0.56), p<0.001$; antipneumococcal penicillin and macrolide 0.81 (0.50-1.34), $p=0.42$. Mortality rates: antipneumococcal cephalosporin, $16.2 \%$; levofloxacin, 9.1\%; antipneumococcal penicillin, 15.9\%; antipneumococcal cephalosporin and macrolide, 5.7\%; antipneumococcal penicillin and macrolide, $12.2 \%$. the more severe patients (Fine class V). This clinical improvement is probably related to treatment choice, as the effect was maintained after correction for confounding factors.

Analysis of the overall population (RP and PP patients) confirmed that the compliance to the guidelines was associated with a significant improvement in terms of success rate and an advantage in terms of mortality, with a faster discharge from hospital. Levofloxacin monotherapy was the most active treatment in terms of success rate, followed by the combination of either levofloxacin or advanced macrolides with antipneumococcal cephalosporins. Moreover, advanced macrolides, in combination with antipneumococcal cephalosporins and levofloxacin monotherapy, were the best treatment approaches in terms of reduction of mortality.

These findings are consistent with retrospective data in the literature indicating that the most successful antibiotic approaches are the combined use of a cephalosporin plus a macrolide, or a fluoroquinolone alone [12-14]. A more recent prospective study limited the survival benefits of combination therapy to more severely ill patients with bacteraemic pneumococcal pneumonia [15]. Similar conclusions were also reached in an article by SHEFET et al. [16].

Interpretation of differences in the outcome of therapeutic approaches in the present study, as well as in similar surveys, should be approached with caution considering that the treatment assignment was not randomised. However, in the current study, efforts were made to adjust outcome analyses for known risk factors (Fine score) and for study centre (a source of variation possibly related to otherwise unspecified confounders). These corrections may be expected to reduce, but not eliminate, possible biases related to baseline differences between patients given different antibiotics. A further bias affecting outcome may be due to underdosing of some antibiotic classes compared with others. This does not appear to be the case in the present study, as drug dosing schemes were in line with guideline indications.

The present study confirms the association between Fine score and mortality, and also indicates a possible role of this scoring 
system in predicting clinical failure. Known risk factors, such as increased age, concomitant diseases and nursing home residence, were included in the Fine scoring and were, therefore, not considered as independent predictors in the analyses.

Another interesting finding of the present study is that previous antibiotic treatment, in the 2 months preceding admission, was associated with clinical failure but not with mortality.

The microbiological data were not reported in detail because an aetiological diagnosis was available in only a very limited number of cases $(<12 \%$ of patients). This is presumably the reason for the extremely divergent data, in terms of leading pathogens, compared with recognised epidemiological data. Most available specimens were sputum samples, with very low rates of blood culture and urinary antigen detection. These findings underscore the need for a better implementation of guideline recommendations regarding aetiological testing in hospitalised pneumonia cases.

In the present study, $>70 \%$ of cases were aged $65-90$ yrs and almost $20 \%$ of patients were aged $\geqslant 90$ yrs. Moreover, $\sim 70 \%$ of patients had two or more chronic diseases associated with CAP, and it is known that age and major comorbidities are among the factors independently associated with mortality in very elderly patients $[17,18]$. A recognised major limitation of the Fine score is the impact of age on the score. Given the high number of elderly patients in the current study population, this may have had a significant impact on the number of Fine class IV and V cases recorded. This may also explain the fact that a relatively high number $(>30 \%)$ of Fine V patients, in both RP and PP, were treated in an IM ward and not admitted to an ICU.

One of the strengths of the present study is the large population of patients enrolled, despite the limitation that half of these patients were retrospectively analysed. Another limitation is the lack of precise identification of changes in other factors, not directly related to antibiotic therapy, which could possibly be linked with the better outcome in the PP. These could include changes in microbiological assessment (such as urinary antigen test use), time-point of antibiotic therapy, and drug dosage. None of these factors changed between the two phases of the study. The main confounding factor during the study period could have been the antimicrobial profile of resistance in pneumococci spp. and Haemophilus influenzae or Moraxella catarrhalis. However, on the basis of Italian data from the Protekt Study [19], resistance to macrolides in Streptococcus pneumoniae and to $\beta$-lactams in $H$. influenzae has increased only slightly from 2000 to 2004 , from 35 to $40 \%$ and from 15 to $20 \%$, respectively. During the same time period, no change in susceptibility to respiratory fluoroquinolone occurred in these respiratory pathogens. In any case, these kinds of change could have had a negative impact on the outcome of monotherapies with macrolide alone, but this effect would account for $<3 \%$ of noncompliant therapies.

At the start of the present study, and according to the existing guidelines, nursing home patients were included as CAP. Being a nursing home resident is acknowledged as a risk factor in the Fine scoring system by assigning 10 additional points. A sensitivity analysis was performed, excluding nursing home patients, which demonstrated that this did not alter the main findings of the study.

The main results of the current study are the demonstration that guidelines can improve CAP management and that guideline implementation through simple educational measures, such as ad hoc investigator meetings, showed sufficient compliance to positively affect patient outcome. Unfortunately, this simple approach is probably insufficient, as overall compliance to guidelines was still quite low during the PP $(44 \%)$. Nonetheless, considering that even this partial improvement in compliance was associated with clinically meaningful positive outcomes, further efforts are required to obtain greater guideline implementation.

It is worth noting that recent studies also reported improvement in clinical outcome measures associated with communityacquired pneumonia treatment following guideline utilisation $[6,7]$. The results of the present study underscore the need for more aggressive or proactive approaches towards implementation, in order to further improve the overall management of community-acquired pneumonia.

\section{ACKNOWLEDGEMENTS}

The authors would like to thank M. Larosa (Medical Direction of Sanofi-Aventis Italia, Milan, Italy) and Studio Associato Airoldi, Cicogna e Ghirri (Milan) for their valuable assistance in designing and performing the statistical analysis.

The FADOI Study on CAP (FASTCAP) study investigators were as follows. P. Agostinelli: Medicina Interna Ospedale di Jesi; A. Bargiggia: Medicina Interna Ospedale. S. Carlo, Milan; F. Bennicelli: Medicina Interna Ospedale di Tortona; A Bulfoni: Medicina II, Azienda Ospedale Univ. Santa Maria della Misericordia di Udine; S. Bergamo: Divisione Medicina Ospedale Civile Este; G. Bertramello: Medicina Generale Ospedale di Bassano del Grappa; F. Casu: Medicina Interna Ospedale S. Margherita Ligure; A. Corvetta: Medicina I Azienda Ospedaliera Rimini; R. Cosentini: Medicina d'Urgenza, Policlinico IRCCS Milano; S. Costantino: Area di Medicina Interna, Università Campus Bio-Medico, Roma; F. D'Amore: Medicina Interna Ospedale Sandro Pertini Roma; A. D'Angelo: Medicina Interna, Ospedale Buccheri La Ferla FBF Palermo; G. Fabio: Medicina Interna Policlinico IRCCS Milano; A. Frasca: Medicina Interna Ospedale di Piedemonte Matese; G. Gulli: Medicina Interna Ospedale Bianchi Melacrino Morelli Reggio Calabria; V. Inserra: Medicina Interna I Ospedale Garibaldi Catania; I. Iori: Dip. di Medicina Interna, Az. Ospedale Arcispedale S. Maria Nuova, Reggio Emilia; M. Lanfredini: Medicina Gen. I Ospedale San Paolo, Milano; G. Lo Pinto: Medicina Interna Ospedale Galliera Genova; F. Miceli: Divisione di Medicina Interna Ospedale Civile Jazzolino Vibo Valentia; F. Montanar: Medicina Interna Palmanova (UD); A. Morettini: Medicina Interna Ospedale Careggi Firenze; C. Nozzoli: Medicina Interna II Ospedale Careggi Firenze: T. Paolini: Medicina Interna II, Ospedale SS. Annunziata Sassari; R. Potì: Medicina Interna Ospedale; V. Fazzi Lecce: Nuovo Ospedale; S. Donato Arezzo, F. Puzzolante and M. Errico: Medicina Interna IRCCS Ospedale C.S. Sofferenza-S.G. Rotondo; V. Russo: Medicina d' Urgenza Ospedale Cardarelli Napoli; F. Torta: Medicina Interna Ospedale di Chieri; A. Trotta: Reparto di Medicina Ospedale San Salvatore, Regionale di Coppito Aquila; 
A. Venco: Medicina I Ospedale di Circolo Varese; D. Vanni Medicina Interna ad Indirizzo Geriatrico Arezzo.

\section{REFERENCES}

1 Macfarlane JT, Colville A, Guion A, Macfarlane RM, Rose DH. Prospective study of aetiology and outcome of adult lower-respiratory-tract infections in the community. Lancet 1993; 341: 511-514.

2 Jokinen C, Heiskanen L, Juvonen H, et al. Incidence of community-acquired pneumonia in the population of four municipalities in eastern Finland. Am J Epidemiol 1993; 137: 977-988.

3 Mandell LA, Wunderink RG, Anzueto A, et al. Infectious Diseases Society of America/American Thoracic Society consensus guidelines on the management of communityacquired pneumonia in adults. Clin Infect Dis 2007; 44: Suppl. 2, S27-S72.

4 Woodhead M, Blasi F, Ewig S, et al. Guidelines for the management of adult lower respiratory tract infections. Eur Respir J 2005; 26: 1138-1180.

5 Fine MJ, Auble TE, Yealy DM, et al. A prediction rule to identify low-risk patients with community-acquired pneumonia. N Engl J Med 1997; 336: 243-250.

6 Menéndez R, Torres A, Zalacain R, et al. Guidelines for the treatment of community-acquired pneumonia: predictors of adherence and outcome. Am J Respir Crit Care Med 2005; 172: 757-762.

7 Dean NC, Bateman KA, Donnelly SM, Silver MP, Snow GL, Hale D. Improved clinical outcomes with utilization of community-acquired pneumonia guideline. Chest 2006; 130: 794-799.

8 Rello J, Bodi M, Mariscal D, et al. Microbiological testing and outcome of patients with severe community-acquired pneumonia. Chest 2003; 123: 174-180.

9 Ewig S, Ruiz M, Mensa J, et al. Severe community-acquired pneumonia. Assessment of severity criteria. Am J Respir Crit Care Med 1998; 158: 1102-1108.
10 Standards for the diagnosis and care of patients with chronic obstructive pulmonary disease (COPD) and asthma. This official statement of the American Thoracic Society was adopted by the ATS Board of Directors, November 1986. Am Rev Respir Dis 1987; 136: 225-244.

11 Blasi F, Bulfoni A, Concia E, et al. Gestione delle infezioni delle basse vie respiratorie in medicina interna [Management of lower respiratory tract infections in internal medicine]. Ital J Intern Med 2002; 1: Suppl. 2, 1-69.

12 Gleason PP, Meehan TP, Fine JM, Galusha DH, Fine MJ. Associations between initial antimicrobial therapy and medical outcomes for hospitalized elderly patients with pneumonia. Arch Intern Med 1999; 159: 2562-2572.

13 Houck PM, MacLehose RF, Niederman MS, Lowery JK. Empiric antibiotic therapy and mortality among medicare pneumonia inpatients in 10 western states: 1993, 1995, and 1997. Chest 2001; 119: 1420-1426.

14 Arnold FW, Summersgill JT, Lajoie AS, et al. A worldwide perspective of atypical pathogens in community-acquired pneumonia. Am J Respir Crit Care Med 2007; 175: 1086-1093.

15 Baddour LM, Yu VL, Klugman KP, et al. Combination antibiotic therapy lowers mortality among severely ill patients with pneumococcal bacteremia. Am J Respir Crit Care Med 2004; 170: 440-444.

16 Shefet D, Robenshtock E, Paul M, Leibovici L. Empiric antibiotic coverage of atypical pathogens for community acquired pneumonia in hospitalized adults. Cochrane Database Syst Rev 2005; 2: CD004418.

17 Fernández-Sabé N, Carratalà J, Rosón B, et al. Communityacquired pneumonia in very elderly patients: causative organisms, clinical characteristics, and outcomes. Medicine 2003; 82: 159-169.

18 Ewig S, Torres A, Woodhead M. Assessment of pneumonia severity: a European perspective. Eur Respir J 2006; 27: 6-8.

19 Marchese A, Ardito F, Fadda G, et al. PROTEKT Italy: third year results and conclusions. G Ital Microbiol Med Odontoiatrica Clin 2005; 9: 69-106. 